IWSCFF 17-07

\title{
FLIGHT DEMONSTRATION OF SPACEBORNE REAL-TIME ANGLES-ONLY NAVIGATION TO A NONCOOPERATIVE TARGET IN LOW EARTH ORBIT
}

\author{
Jean-Sébastien Ardaens* and Gabriella Gaias ${ }^{\dagger}$
}

\begin{abstract}
The paper presents the flight performance of the onboard vision-based navigation software employed during the AVANTI experiment. Two autonomous rendezvous to a noncooperative object have been performed in orbit, first from $13 \mathrm{~km}$ to $1 \mathrm{~km}$ separation, then from $3 \mathrm{~km}$ to $50 \mathrm{~m}$. Despite the poor visibility conditions and the strong orbit perturbations encountered at low altitude, the onboard filter was able to support the onboard guidance and control algorithms with accurate and reliable relative state estimation, enabling thus a safe and smooth approach.
\end{abstract}

\section{INTRODUCTION}

In-orbit technological realizations always constitute a priceless mine of lessons learnt and valuable experience, and the AVANTI (Autonomous Vision Approach Navigation and Target Identification) demonstration is no exception. This experiment was successfully conducted by the German Aerospace Center (DLR/GSOC) in autumn 2016 and could demonstrate spaceborne autonomous rendezvous to a noncooperative target using solely line-of-sight measurements. ${ }^{1}$

The spaceborne algorithms, constituting the core of the experiment, have been embedded as passenger software on BIROS, a German Earth observation satellite launched in June 2016 as part of the FireBird constellation. ${ }^{2}$ This choice was motivated by the fact that this spacecraft was carrying a third-party picosatellite (BEESAT- $4^{3}$ ) to be released in orbit using a dedicated ejection mechanism, ${ }^{4}$ which means that an appealing target was generated for free to support the experiment without the need of spending propellant to fly to an existing object. In addition, BIROS could grant access to the key hardware devices required by the experiment: a camera and a propulsion system. No additional formation-flying sensors or actuators were embarked, so that the entire experiment had been designed to use one of the star cameras as unique sensor to track the target object.

AVANTI has been designed relying on the experience already collected in 2012 using the PRISMA formation flying testbed. At that time, the so-called ARGON (Advanced Rendezvous demonstration using GPS and Optical Navigation ${ }^{5}$ ) experiment had already tackled the problem of angles-only relative navigation by exercising a ground-in-the-loop approach to a noncooperative target using optical methods. Compared to this precursor experiment, AVANTI presents an increased level of complexity to cope with a more realistic scenario, in view of the future possible applications of such a technological know-how: rendezvous to space debris or to a noncooperative satellite to be serviced. ${ }^{6}$

\footnotetext{
*German Space Operations Center (DLR/GSOC), 82234 Wessling, Germany.
}

${ }^{\dagger}$ German Space Operations Center (DLR/GSOC), 82234 Wessling, Germany. 
One of the major advances brought by AVANTI is summarized by the first letter of the experiment name: Autonomy. For this purpose a real-time onboard navigation filter is needed to support the guidance and control algorithms with accurate estimate of the state of the formation. As sketched out in the first section, the navigation filter employed by AVANTI processes the line-of-sight measurements to the target spacecraft, which are previously extracted by a dedicated target detection algorithm from the images collected onboard using one of the star cameras of the satellite. It has to be emphasized that the experiment has been conceived to deal with a truly uncooperative target, relying only on pictures to estimate precisely the state of the formation. As a matter of fact, the images collected in orbit were really the only available observations, since the GPS receiver embarked by BEESAT $-4^{7}$ was unfortunately not yet operational during the time slot allocated to AVANTI.

Designing a reliable and accurate angles-only real-time navigation software is a challenging task. Extensive analyses had been conducted during the development of the onboard filter using an advanced simulation environment. ${ }^{8}$ However the experimental conditions were even worse than the simulated worst case. The paper addresses specifically the lessons learnt which have been collected during the experimental timeline. Overall the weak observability of line-of-sight navigation has been a recurrent issue throughout the whole experiment. This problem is of course well-known as well as the solution to improve it, which consists in executing calibrated maneuvers to change the formation configuration over time. ${ }^{9}$ As explained in the second section, three additional major obstacles have been constantly contributing to degrade the observability, making the life of the filter pretty difficult: a poor visibility, an unknown time-varying differential drag, and large maneuver execution errors.

Despite all these difficulties, the paper shows that the filter design retained for AVANTI was perfectly suited for the needs of the experiment. Two approaches have been performed autonomously: from $13 \mathrm{~km}$ to $1 \mathrm{~km}$ (19-23 November 2016) and from $3 \mathrm{~km}$ to $50 \mathrm{~m}$ (25-28 November, 2016). ${ }^{1}$ Initialized from the ground with a reasonably good guess of the relative state, the filter was able to support the onboard controller with a navigation solution accurate at the meter level in the lateral direction and to about $10 \%$ of the intersatellite separation in the boresight direction. In the absence of other sensors to evaluate independently the state of the formation, the filter performance has been assessed using precise relative orbit determination products generated on ground using the same set of data. ${ }^{10}$ Thanks to more advanced and resource-consuming processing techniques (precise estimation of the maneuver execution errors using raw GPS data, implementation of a batch least-squares estimator, advanced data screening), these products were more robust and were thus considered as reference throughout the mission to monitor the behavior of the onboard navigation.

\section{SYSTEM OVERVIEW}

The design of the onboard relative navigation filter has been already described in Reference 8, which should serve as baseline to the Reader for further details. The main characteristics are recalled here to ease the interpretation of the flight results presented in the sequel.

The relative navigation task consists in providing continuously an estimate of the state of the formation in real-time to the other onboard applications (guidance, control, attitude pointing). Since the star trackers are the only sensors available for relative navigation, this estimation is derived from line-of-sight measurements which have to be first extracted from the images taken by the camera. Because of some hardware limitations of the host satellite, ${ }^{1}$ the pictures are taken only every $30 \mathrm{~s}$. Figure 1 depicts the resulting system architecture: a target detection module is in charge of recognizing the desired object in the pictures, and provides the resulting angles-only measurements 
to a dynamical filter. The latter processes these observations and, thanks to the knowledge (provided externally) of the absolute state and maneuvers executed by the chaser spacecraft (i.e, BIROS), derives the relative state estimate.

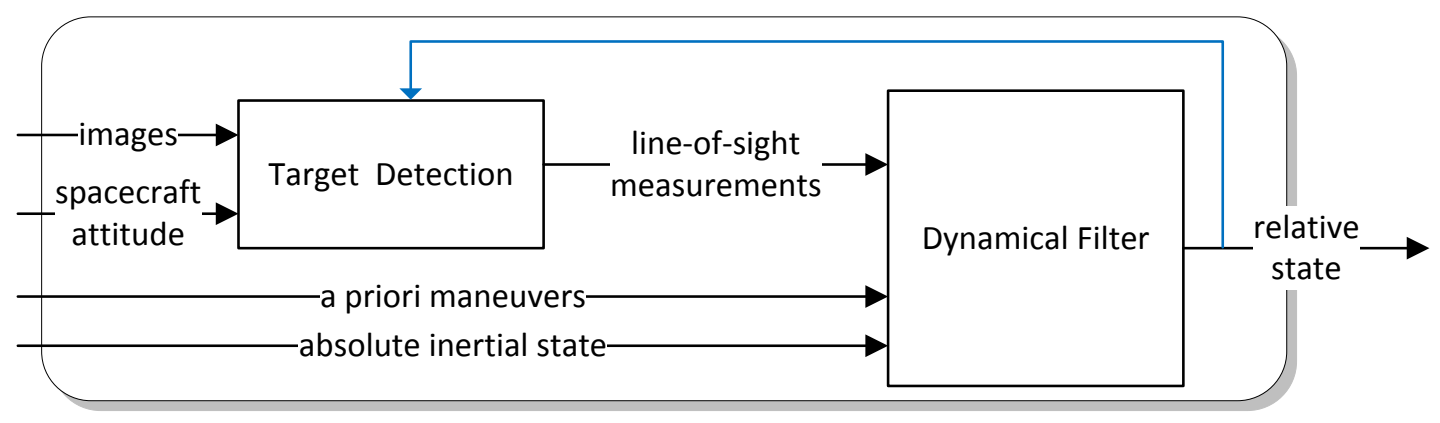

Figure 1. Functional view of the relative navigation system

The target detection module makes uses of a star catalog to distinguish the stars in the image background from non-stellar objects. In order to limit the computational load, the coarse knowledge of the orientation of the star tracker (derived from the onboard attitude) is used to identify the stars. A reliable detection of the target at far-range is not a trivial task, since its poor luminosity does not allow for an obvious recognition and since many plausible candidate objects might be simultaneously visible. Consequently, a dedicated algorithm has been designed to recognize an object flying on a similar orbit, based on its apparent trajectory which differs greatly from the trajectories of other parasite bodies. ${ }^{8}$ This technique is named in the sequel kinematic target detection. It has been later recognized that, once the filter has converged, the relative state information can also be of great help detecting the target. As a result, compared to the design presented in Reference 8, an additional feedback has been introduced in the flight software (blue arrow in Figure 1) to ease the recognition in case that the filter is already locked to a valid solution. This feature is named in the sequel dynamic target detection.

The onboard filter is based on a special parametrization of the relative motion, described by a set $\boldsymbol{\delta} \boldsymbol{\alpha}$ of dimensionless relative orbital elements: ${ }^{11}$

$$
\boldsymbol{\delta} \boldsymbol{\alpha}=\left(\begin{array}{llllll}
\delta a & \delta e_{x} & \delta e_{y} & \delta i_{x} & \delta i_{y} & \delta u
\end{array}\right)^{T},
$$

where $\delta a$ is the relative semi-major axis, $\delta \boldsymbol{e}=\left(\delta e_{x}, \delta e_{y}\right)^{T}$ and $\delta \boldsymbol{i}=\left(\delta i_{x}, \delta i_{y}\right)^{T}$ are called respectively relative eccentricity and inclination vectors, and $\delta u$ stands for the relative mean argument of latitude. The relative orbital elements are used to describe the state of the formation and can easily be translated in a Cartesian representation if needed. The in-plane relative motion is described by $\delta a, \delta \boldsymbol{e}$ and $\delta u$ whereas $\delta \boldsymbol{i}$ is responsible for the cross-track motion. Note that $\delta u$ is sometimes equivalently replaced in the literature by the relative mean longitude $\delta \lambda=\delta u+\operatorname{cotan}(i) * \delta i_{y}$, where $i$ represents the orbit inclination.

As already emphasized in the past, ${ }^{12}$ this parametrization is of great interest when dealing with formation of satellites, since it offers a quick insight into the geometry of the relative motion and a simple criteria to guarantee the safety of the formation (using a proper phasing of the relative eccentricity and inclination vectors). For embedded onboard systems, its major advantage lies rather in the associated analytical dynamical model, which provides an accurate and computationallylight prediction of the relative motion thanks to the inclusion of the perturbation due to the Earth's 
oblateness $\left(J_{2}\right)$. Mathematically, the model relates the state $\boldsymbol{\delta} \boldsymbol{\alpha}$ of the formation at time $t$ to the state at epoch $t_{0}$ by the means of the state transition matrix $\mathbf{\Phi}$ :

$$
\boldsymbol{\delta} \boldsymbol{\alpha}(t)=\boldsymbol{\Phi}\left(t-t_{0}\right) \cdot \boldsymbol{\delta} \boldsymbol{\alpha}\left(t_{0}\right)
$$

This analytical model has been notably successfully employed to design the previous DLR's spaceborne autonomous formation-flying experiments implemented on the TanDEM- $\mathrm{X}^{13}$ and PRISMA ${ }^{14}$ satellites. As far as AVANTI is concerned, the formation undergoes however a much stronger differential drag, due to its low altitude $(500 \mathrm{~km})$ and to the very different ballistic coefficients of BIROS and BEESAT-4. As a result, the relative motion model has been revisited during the design of the AVANTI experiment to refine the modeling of the disturbance due to $J_{2}$ and to introduce in addition the mean effects of the differential drag. ${ }^{15}$ Since this latter perturbation is extremely difficult to be modeled accurately, it has been decided for simplicity to make use of external empirical values describing the resulting time variations of $\delta a$ and $\delta \boldsymbol{e}$, which are approximated to constant values. The refined dynamical model takes thus as input three additional parameters $\left(\delta \dot{a}, \delta \dot{e}_{x}, \delta \dot{e}_{y}\right)$ and becomes:

$$
\boldsymbol{\delta} \boldsymbol{\alpha}(t)=\boldsymbol{\Phi}\left(t-t_{0}, \delta \dot{a}, \delta \dot{e}_{x}, \delta \dot{e}_{y}\right) \cdot \boldsymbol{\delta} \boldsymbol{\alpha}\left(t_{0}\right)
$$

The autonomy of the system would however suffer from the external manual provision of these additional parameters. As a consequence, they have to be estimated onboard. The weak observability of the problem makes however dangerous the estimation of too many additional parameters, which would endanger the robustness of the solution. As a result, it has been decided to estimate only $\delta \dot{a}$, neglecting thus the impact of the differential drag on $\delta \boldsymbol{e}$ (which is anyway smaller than the one on $\delta a$. The state vector describing the formation which has to be estimated onboard can thus be written as:

$$
\boldsymbol{x}=\left(\begin{array}{ll}
\boldsymbol{\delta} \boldsymbol{\alpha}^{T} & \delta \dot{a}
\end{array}\right)^{T},
$$

In view of the real-time requirements, an Extended Kalman Filter (EFK) as been chosen as estimator. The time update at new epoch $t$ is done according to Eq. 3, whereas the filter measurement update is done using the line-of-sight measurements of the target object. ${ }^{8}$ Compared to other estimation techniques (for instance least-squares adjustment), the Kalman filter offers the advantage of using process noise to cope with the errors of the dynamical model. This is of great importance since, in addition to the errors due to the differential drag, the filter has to cope with maneuver execution errors. On the other hand, the improvement of observability is obtained by considering the effect of maneuvers over time, requiring thus some filter memory, which fades quickly when introducing too much process noise. A tradeoff needs thus to be found between this contradictory statements. The following filter settings, derived from extensive simulations, were used during AVANTI experiment (note how the values differ between the relative orbital elements to better reflect the anisotropy of the problem):

Table 1. Adopted filter settings

\begin{tabular}{c|c}
\hline Item & Value \\
\hline $\begin{array}{c}\text { Process-noise covariance matrix } \\
\text { Measurement noise }\end{array}$ & diag(1e-8,1e-3,1e-3,1e-3,1e-3,1e-2,1e-8,1e-14) \\
80 arcsec \\
\hline
\end{tabular}




\section{THE AVANTI EXPERIMENT}

The conduction of the AVANTI experiment gave the opportunity to perform the ultimate flight validation of the relative navigation system. Starting from the in-orbit separation of the BEESAT-4 on September 9, 2016, two months in orbit were necessary for the successful completion of the experiment, most of the time being dedicated to a thorough commissioning of the spacecraft. Dealing with spaceborne autonomous close-proximity formation-flight, it was indeed necessary to ensure that all subsystems involved in the experiment were working properly before starting an autonomous approach. Once the satellite was commissioned, the full featured experiment could start on November 19, 2016, during which two autonomous rendezvous were performed at different characteristic ranges. Figure 2 depicts the intersatellite separation during the two autonomous approaches.

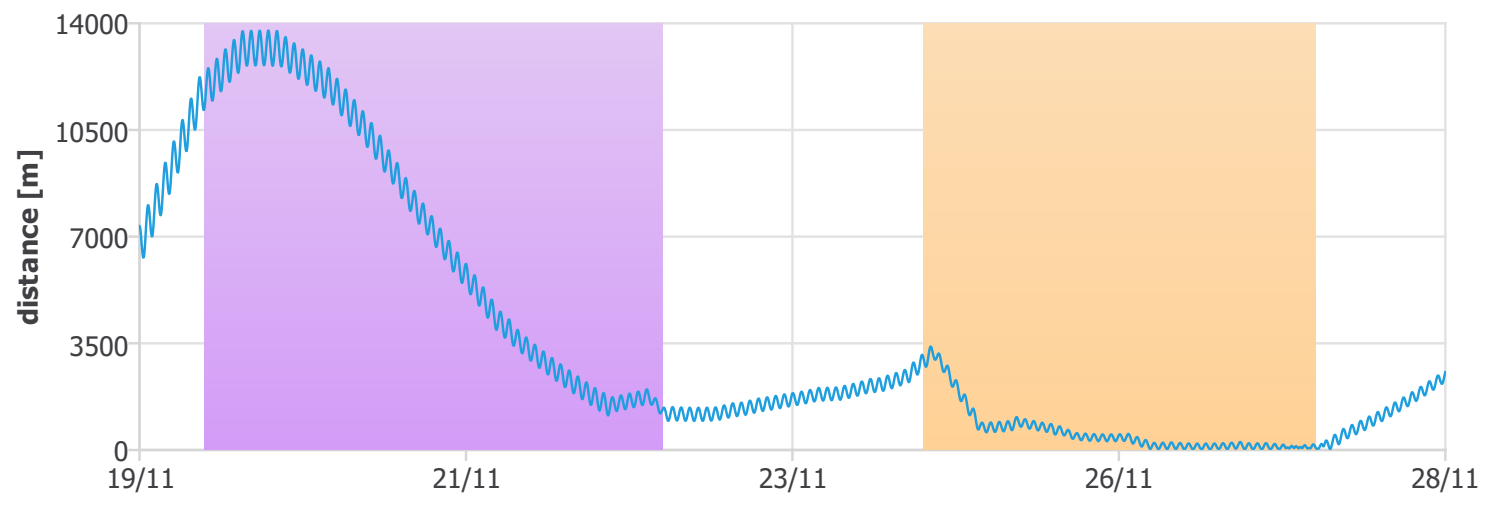

Figure 2. Two autonomous approaches: far to mid range (purple) and mid to close range (orange)

The attentive Reader will have immediately noticed that the distance between the satellites is affected by a sinusoidal pattern. This is due to the fact that, for safety reasons, the approach has not been executed as a simple v-bar approach but in a spiraling fashion as shown in Figure 3. This peculiar relative motion is the consequence of the adopted passive formation safety concept which states that a minimum intersatellite distance will be guaranteed at any time, provided that $\delta \boldsymbol{e}$ and $\delta \boldsymbol{i}$ are properly phased. ${ }^{16}$ The onboard autonomous guidance will have the task of reducing step by step the size of the spiral during the approach and of keeping the desired phasing of the relative eccentricity and inclination vectors.

The numerous simulations conducted during the development phase had shown the pertinence of the adopted design of the relative navigation system. However, it was also obvious that the acid test for the relative navigation task would come when facing the arduous experimental conditions. Among the numerous challenges and constraints encountered during AVANTI, the navigation had to face specifically the following difficulties which were difficult to simulate faithfully prior to the start of the experiment:

- Contrary to ARGON which, thanks to the dusk-dawn orbit of PRISMA, benefited from optimal illumination conditions, AVANTI deals with target objects flying on any kind of low Earth orbits. This has dramatic impacts in terms of visibility, since on one hand the target object is eclipsed during a large part of the orbit and on the other hand the camera becomes blinded by the Sun during another large part of the orbit. As a result, only a tiny portion of the relative motion is visible. Figure 3 provides a clear illustration of the poor visibility 


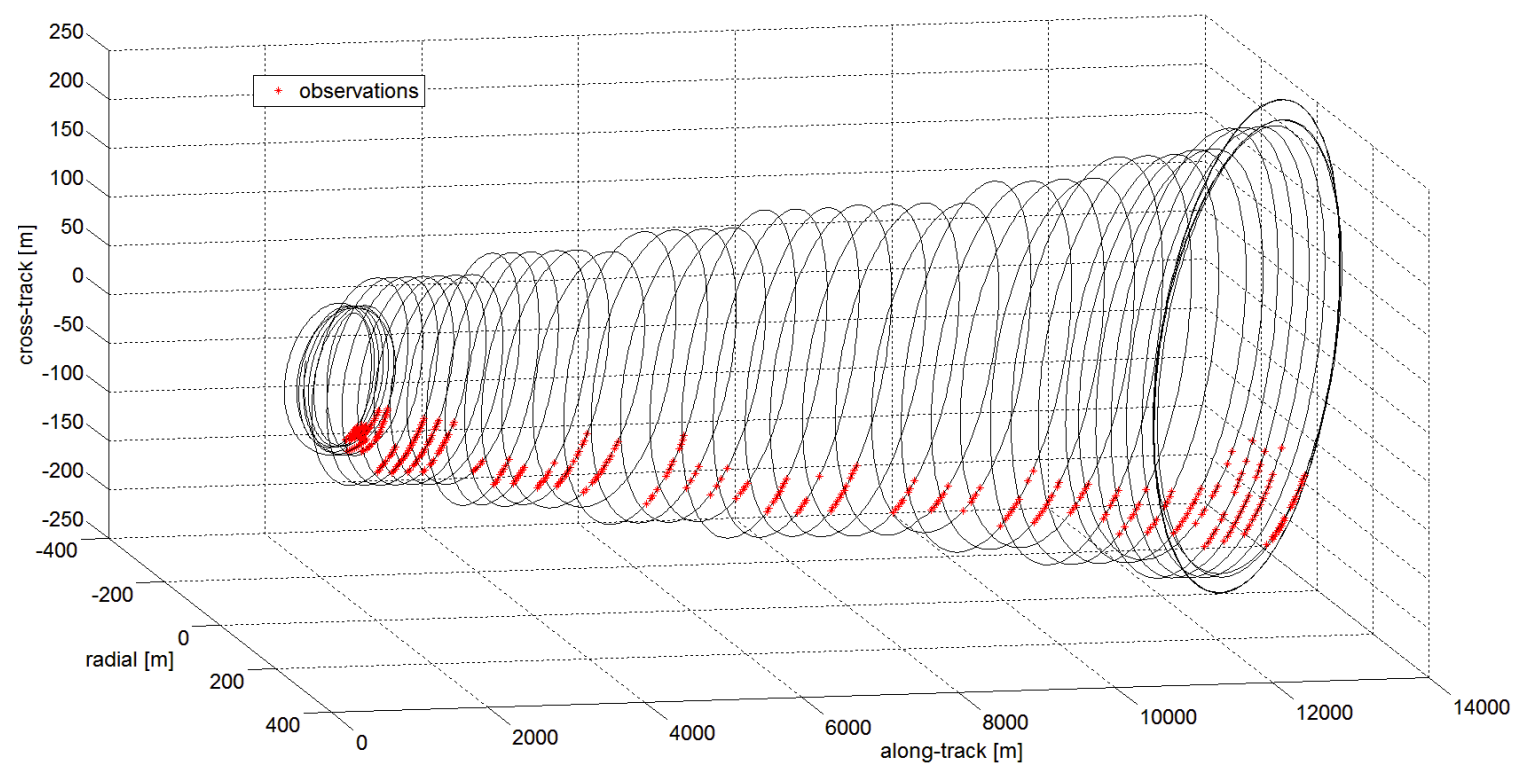

Figure 3. Spiraling approach during the first autonomous approach

conditions, where the red stars represent the line-of-sight measurements which were available during the approach.

- BIROS is equipped with one single cold-gas thruster which needs to be oriented and kept in the proper direction for a long time depending on the size of the maneuver. The BIROS attitude controller was not always able to keep precisely enough the desired attitude, resulting in large maneuver execution errors which added even more uncertainties to the onboard relative motion model.

- Finally, BIROS flies at a low altitude $(500 \mathrm{~km})$ inducing a strong unknown differential drag which has to be estimated as part of the navigation process. The simulations had indicated that this would be possible, under the assumption that the unknown differential drag would have a similar pattern over large time scales.

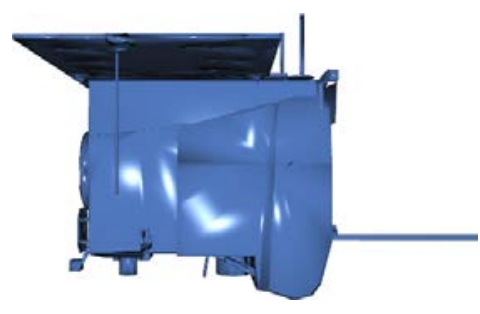

(a) Earth-pointing

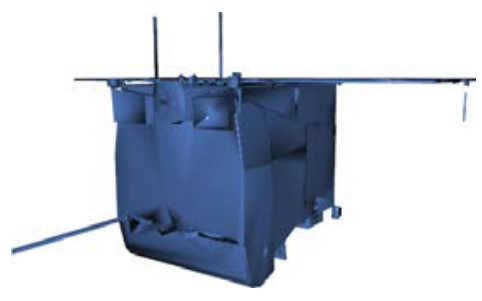

(b) Target-pointing

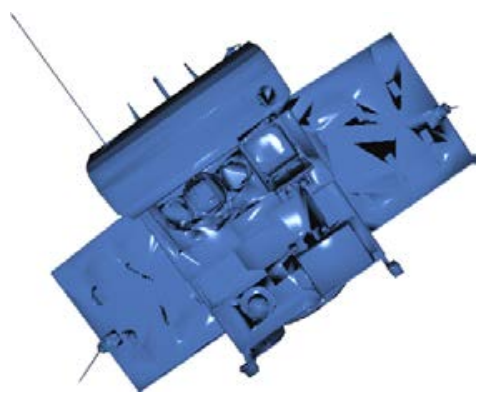

(c) Cooling down

Figure 4. Cross-sectional area for different attitude modes

This assumption revealed itself to be wrong in view of the frequent switches of attitude mode, 
resulting in large variations of the spacecraft cross-sectional area (as shown in Figure 4) and in dramatic changes of the differential drag acting on the satellite. During the experiment, it was often necessary to point the antennas to the ground stations for high-speed data transmission, to orient the thruster to the desired direction of the maneuvers and to enter a special satellite cool-down attitude to cope with unexpected thermal problems. ${ }^{1}$ At mid to close range, these attitude variations were even worse due to the spiraling approach, as seen in the next section.

\section{FLIGHT RESULTS}

\section{Far to Mid Range Autonomous Approach}

The scenario adopted for the first autonomous approach corresponds to the case in which a coarse orbit phasing has been already performed by the ground segment, based on the knowledge of the Two-Line Elements (TLEs) of the target. In view of the poor accuracy of the TLEs, a typical safe distance of more than $10 \mathrm{~km}$ has been kept during this phase. If the initial orbit phasing has been properly done, the size of the relative motion has been already shrunk enough to be able to observe it completely by simply pointing the camera in flight direction.

As already mentioned, the main difficulty at this distance lies in recognizing properly the target. The TLEs are of little help, since their large cross-track error (up to a few hundred meters) does not reduce significantly the target search area in the image, so that many candidates can be simultaneously visible. A kinematic trajectory analysis is thus the preferred way for the target detection when initiating the approach, which is however not a trivial activity considering the few available pictures. Figure 5 depicts the difficulty of this task by focusing on the first hours after the start of the autonomous rendezvous. In the Figure, the total number of centroids visible in the images are drawn in blue, while the centroids which have not been identified as stars are shown in red. Here again, the limited number of measurements is clearly visible: the gray areas represent the eclipse phases, during which the target is not visible, and the remaining areas without centroids correspond to the phases where the camera was blinded by the Sun.

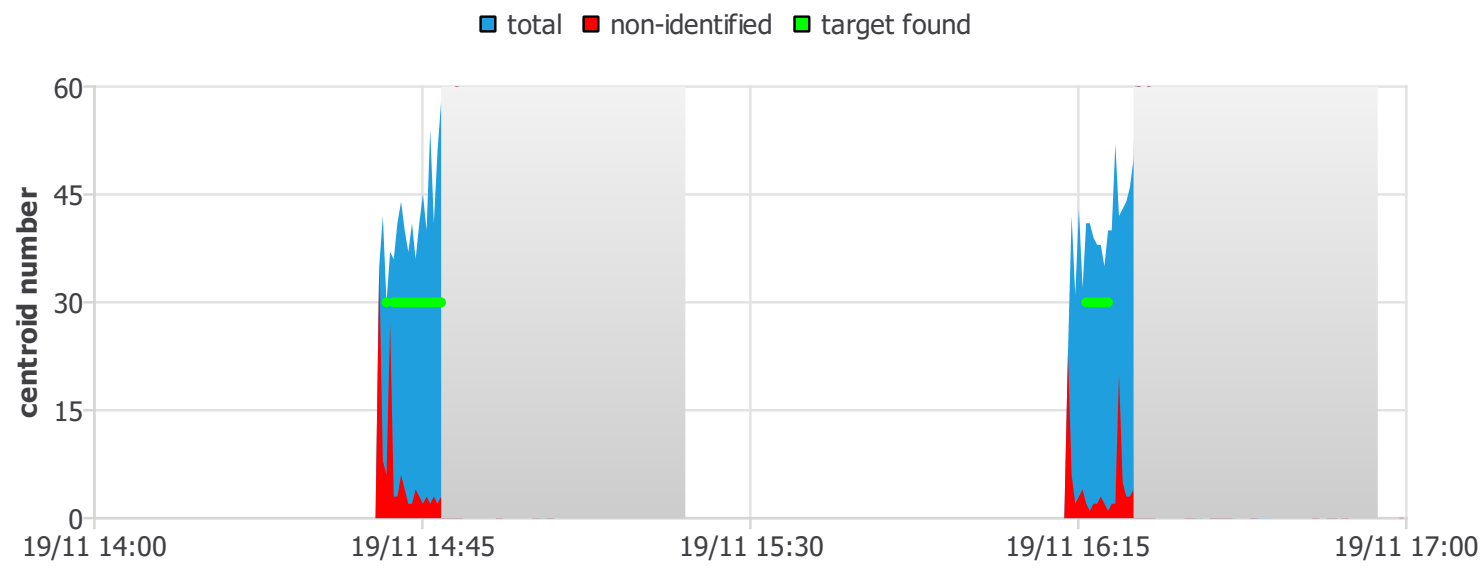

Figure 5. Centroids detected and identified at $13 \mathrm{~km}$ distance

As a result, only approximately 10 minutes of pictures are remaining every orbit, during which the target has to be identified. Figure 5 shows that the number of unrecognized objects is slightly greater than one, with some unexpected peaks where the satellite onboard attitude estimation is 
too degraded to allow for the proper detection of the stars. Nevertheless, despite these anomalies, the strategy adopted for target detection was robust enough to detect successfully the picosatellite (green dots in Figure 5). Note that the detection is not immediate, since the algorithm needs first to collect a sequence of images to be able to detect a candidate trajectory.

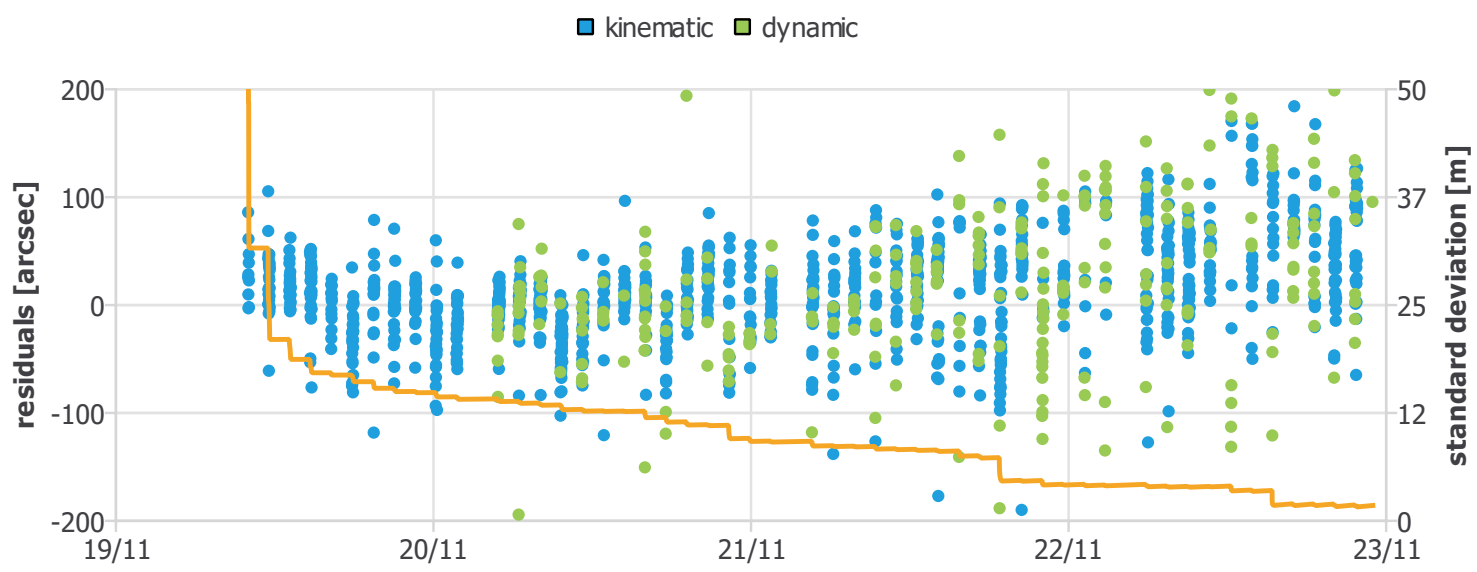

Figure 6. Filter residuals

Once a few line-of-sight observations are successfully extracted from the images, the filter refines its coarse a priori knowledge of the relative state, until it gains enough confidence about the validity of its solution (it is considered that the filter has converged if the standard deviation of the solution decreases under a user-defined threshold). From this moment, the filter state can be used to support the target detection, providing thus more measurements. This behavior is illustrated in Figure 6, where the filter residuals have been plotted during the complete approach. Note how new measurements gained with a dynamic detection appear (in green) once the standard deviation decreases under $13 \mathrm{~m}$ (the standard deviation has been computed excluding $a \delta u$ to provide a measure of the lateral accuracy). A clear degradation of the residuals depending on the distance can be observed (the intersatellite separation decreases continuously during the approach, cf. Figure 2), this aspect will be ever more pronounced at close range and will be treated in the next section

The filter has been initialized with an a priori state $\boldsymbol{\delta} \boldsymbol{\alpha}=\left(\begin{array}{llllll}-50 & 0 & 250 & 0 & 3000 & 10000\end{array}\right)^{T}$ at epoch $t_{0}=2016 / 11 / 19$ 9:00 UTC, which was affected by errors of a few dozen meters for all components (except $a \delta u$ whose error amounted to several hundred meters). Note that this initialization is pretty optimistic and does not really reflect the uncertainties of the TLEs (which could amount to several hundred meters in the worst case scenario). This choice has been motivated by the preliminary analyses of the commissioning phase, which showed that large initialization errors could cause a filter divergence in case of sparse measurements. As a result, the support of a more robust onground vision-based batch least-square filter had been requested to initialize the filter with a more accurate value. Note that this additional aid does not really reduce the value of onboard autonomy, since this preliminary activity could be done for example as part of the coarse orbit phasing.

Overall, despite the sparse measurements, the filter convergence was achieved after a few orbits as depicted in Figure 7. Due to the anisotropy of the problem, the resulting accuracy differs greatly among the orbital elements, especially for $a \delta u$ whose error amounts to several hundred meters at $10 \mathrm{~km}$ distance and diminishes to a few dozen meters at the end of the approach. The lateral accuracy 

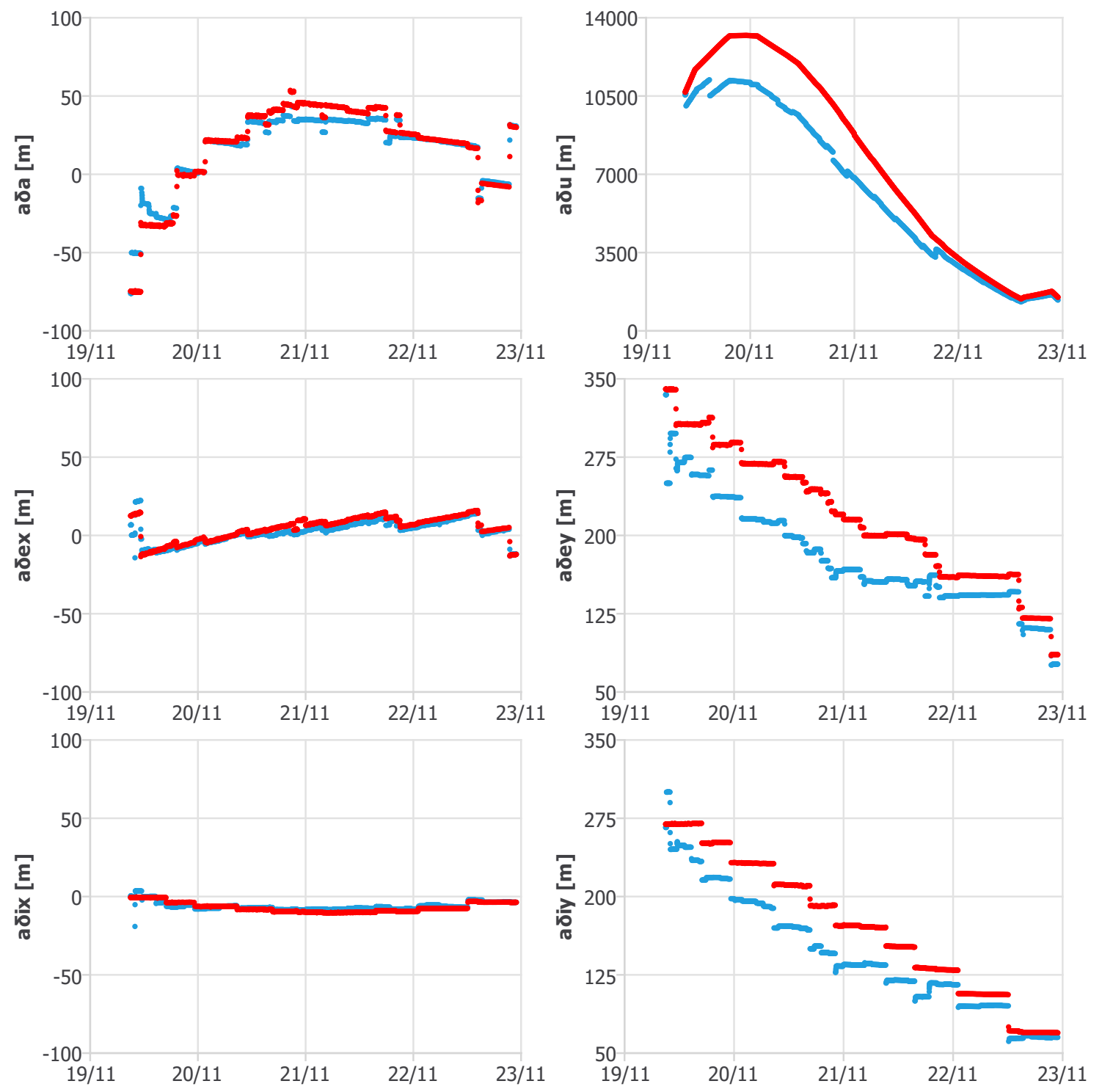

Figure 7. Onboard navigation solution (blue) vs. relative orbit determination (red)

is instead much better: $a \delta a$ is accurate to the meter level (ensuring thus a smooth approach) while the relative eccentricity and inclination vectors are accurately known a few orbits after the start of the approach to about $10 \%$ of their size. The beauty of this concept lies in the fact that the shape of the apparent motion can be estimated accurately in the early stage of the rendezvous, allowing thus the establishment of a safe spiraling approach based on the proper phasing of $\delta \boldsymbol{e}$ and $\delta \boldsymbol{i}$.

It has to be here again emphasized how challenging is the task of the filter in the presence of strong errors affecting the dynamical model and dealing with a poor visibility. Figure 8 summarizes the main sources of errors. Figure 8(a) depicts the maneuver execution errors (assessed post-facto on-ground using precise GPS-based orbit determination). It can be observed that undesired maneuver errors up to $6 \mathrm{~mm} / \mathrm{s}$ were encountered during the experiment value, which is a tremendous value while dealing with precise formation-flying! Figure 8(b) focuses instead on the unmodeled differen- 
tial drag due to the variations of the cross-sectional area. This area has been reconstituted post-facto using attitude data and is associated to the left axis of the plot. Note how this value can vary as much as $100 \%$ during the cool-down phases corresponding to the noticeable blue peaks (cf. Figure 4(c)). The cross-sectional area interacts with the atmosphere density, which varies as well substantially along the orbit (day-night variations), to create a differential drag force. The mean value (over one orbit) of the differential drag force - computed also post facto using a simple Harris-Priester model and assuming an identical drag coefficient for both spacecraft - is plotted in green (associated to the right axis of Figure 8(b)).

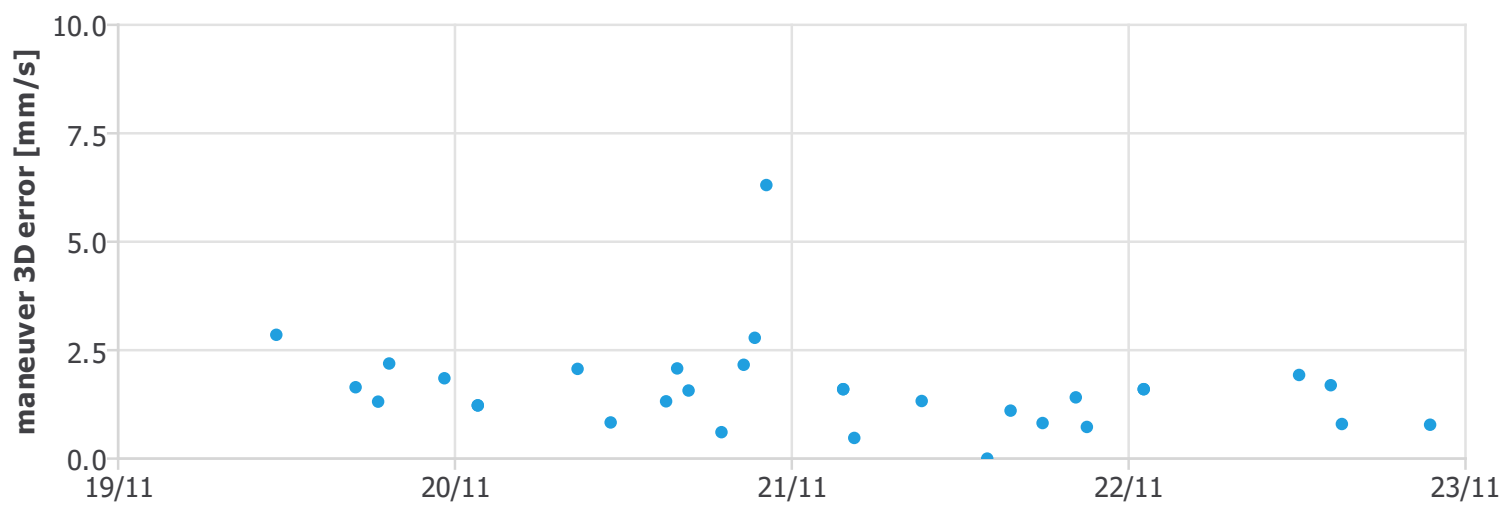

(a) Maneuver errors

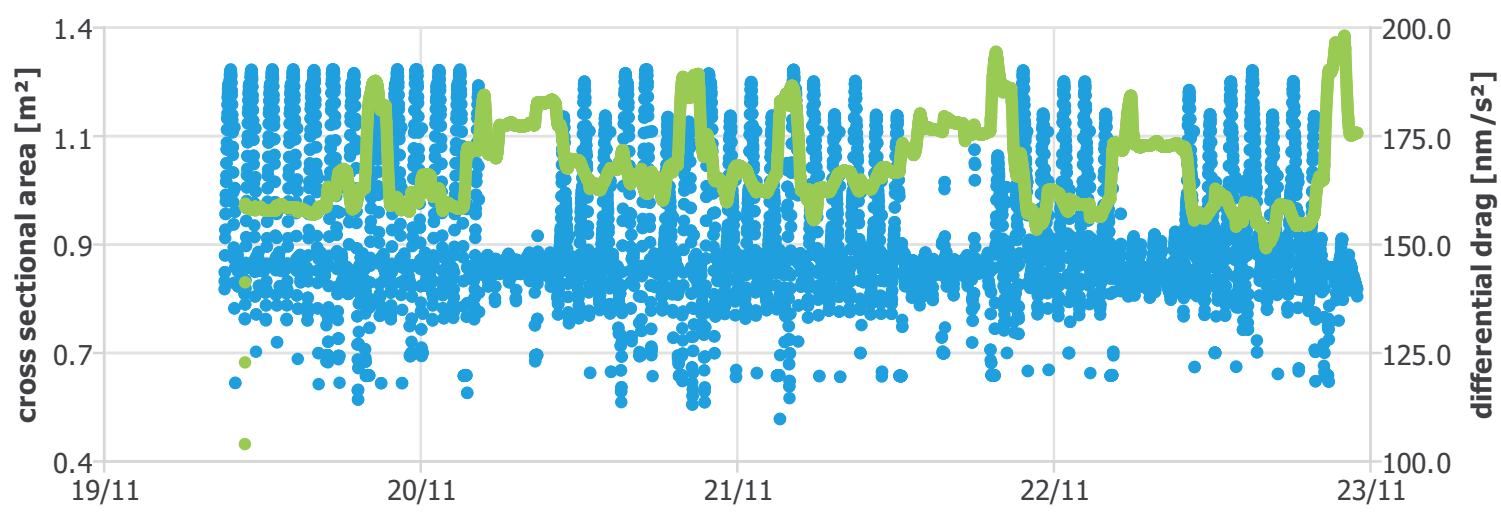

(b) Differential drag

Figure 8. Main errors of the onboard dynamical model

The filter is designed to estimate $a \delta \dot{a}$, that is, the mean effect of this differential drag on the relative semi-major axis. This value is depicted in Figure 9. Given the small number of measurements and the the weak observability, it seems however that this task is too demanding for the filter. A quick look on Figures 8(b) and 9 indicates in fact that the filter is not able to follow these unexpected rapid changes of differential drag, inducing thus errors in the dynamical model which have to be compensated with process noise. This constitutes an important lesson learnt for future similar applications. ${ }^{17}$ In order to improve the response of the filter, a better onboard modeling of the attitude-dependent differential drag might be necessary. This aspect will be investigated in a future work as part of the experiment post-analysis activities. 


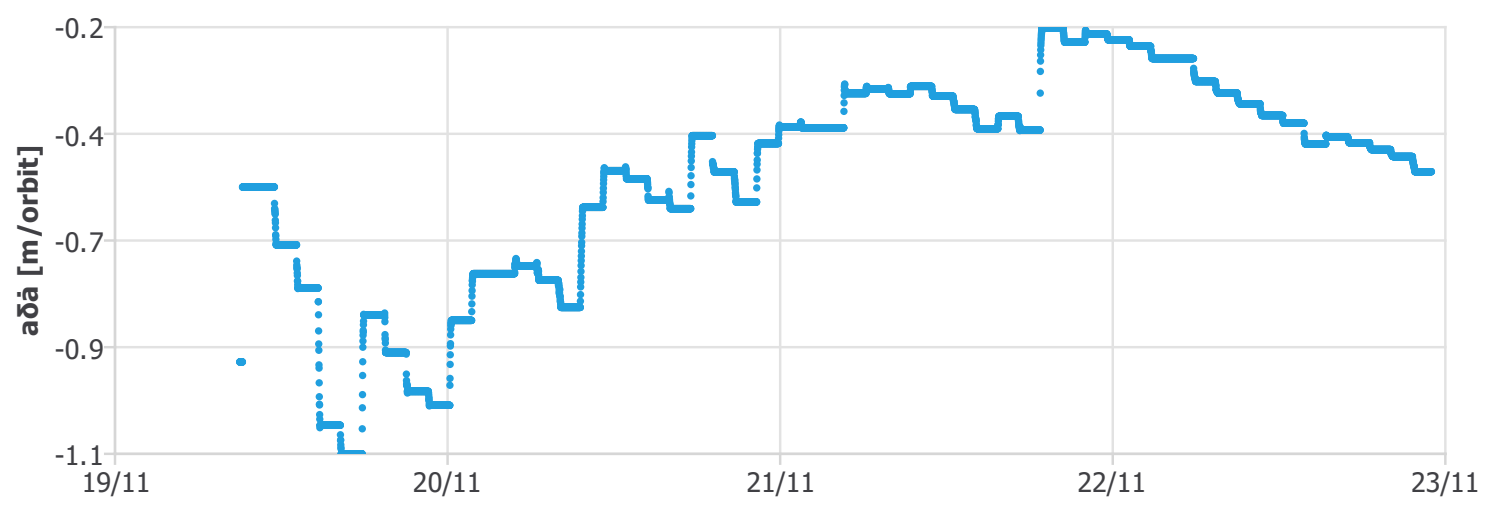

Figure 9. Estimated decay of relative semi-major axis

\section{Mid to Close Range Autonomous Approach}

The AVANTI relative navigation system had been primarily designed to support far to mid range approach. In view of the good system performance observed during the first autonomous approach, it has been decided to investigate to which extend such a filter could reliably work. This triggered the conduction of the second autonomous approach, in order to explore the limits of line-of-sight navigation. At close range, the navigation task becomes much more challenging due to the following reasons:

- When the distance decreases, the errors of the centroids (which should correspond to the center of mass) increase since the target is not imaged as a point anymore, so that the errors of the line-of-sight measurements is larger (as already observed in Figure 6).

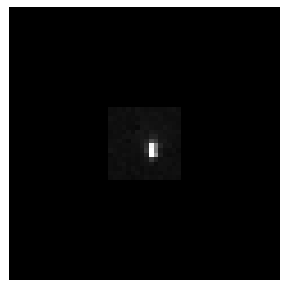

(a) $12 \mathrm{~km}$

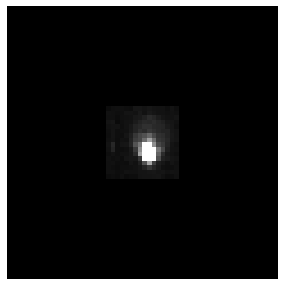

(b) $5 \mathrm{~km}$

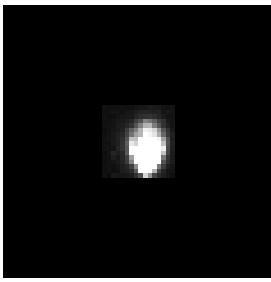

(c) $1 \mathrm{~km}$

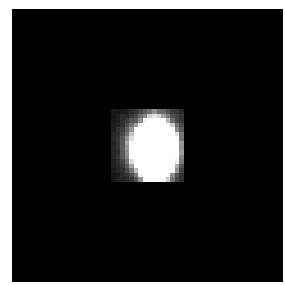

(d) $540 \mathrm{~m}$

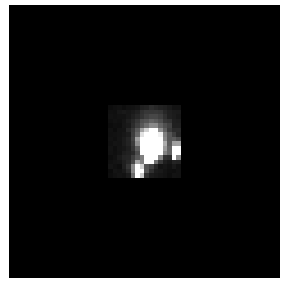

(e) $95 \mathrm{~m}$

Figure 10. Target image at different distances

- At a certain point, the increasing brightness of the target object makes mandatory the use of an electronic shutter, in order to limit the exposure time. The collateral damage is that the stars are not visible anymore in the background, making impossible a precise measurement of the orientation of the camera. As a consequence, the onboard filter has to rely on the onboard attitude estimation to determine the direction of the camera. In the case of AVANTI, the onboard attitude estimation could however not always rely on star trackers, since one head was already blinded by the target object, and the other could not always be oriented to deep sky. As a result, the onboard attitude error was sometimes affected by errors up to one degree (cf. Figure 11)! This is another important lesson learnt: ${ }^{17}$ a similar mission dealing with close-proximity should ensure that at least one star tracker is always working, for example 
by using a third camera head (which was not available in AVANTI). However, it has to be kept in mind that one degree line-of-sight measurement error does not have the same impact at $100 \mathrm{~m}(1.7 \mathrm{~m})$ as at $40 \mathrm{~km}(700 \mathrm{~m})$. It is thus possible to cope with this error by tuning the filter measurement noise (cf. Table 1). In fact, during the close approach, this value has been changed on 25 November 15:00 UTC from 80" (corresponding to 1 pixel) to 1000" in view of the poor onboard attitude estimation performance.

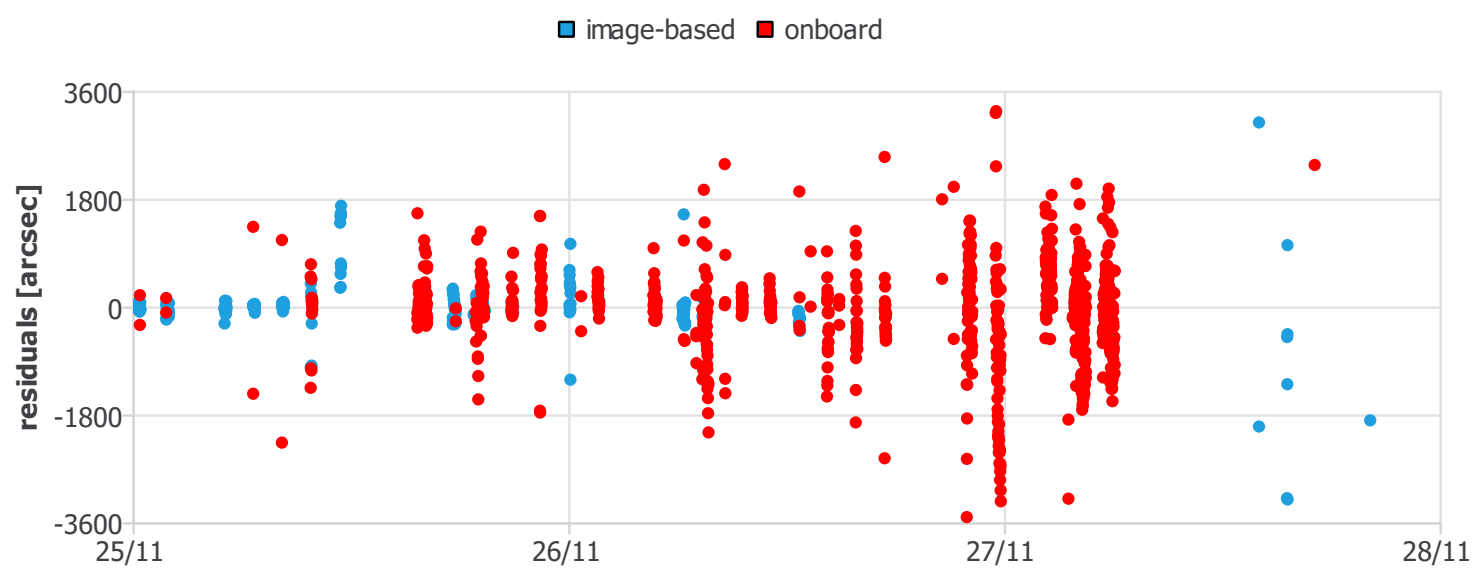

Figure 11. Filter residuals using image-based vs. onboard attitude estimation

- At close range, the cross-sectional area subject to the differential drag suffers from additional variations, in order to follow the target flying on a spiraling relative motion. This effect was limited at far to mid range (the camera head was more or less pointing in flight direction), but become a substantial problem at mid to close range, where the pointing direction can have a large cross-track component. As depicted in Figure 12, the differential drag becomes very different from the values observed previously (Figure 8(b)).

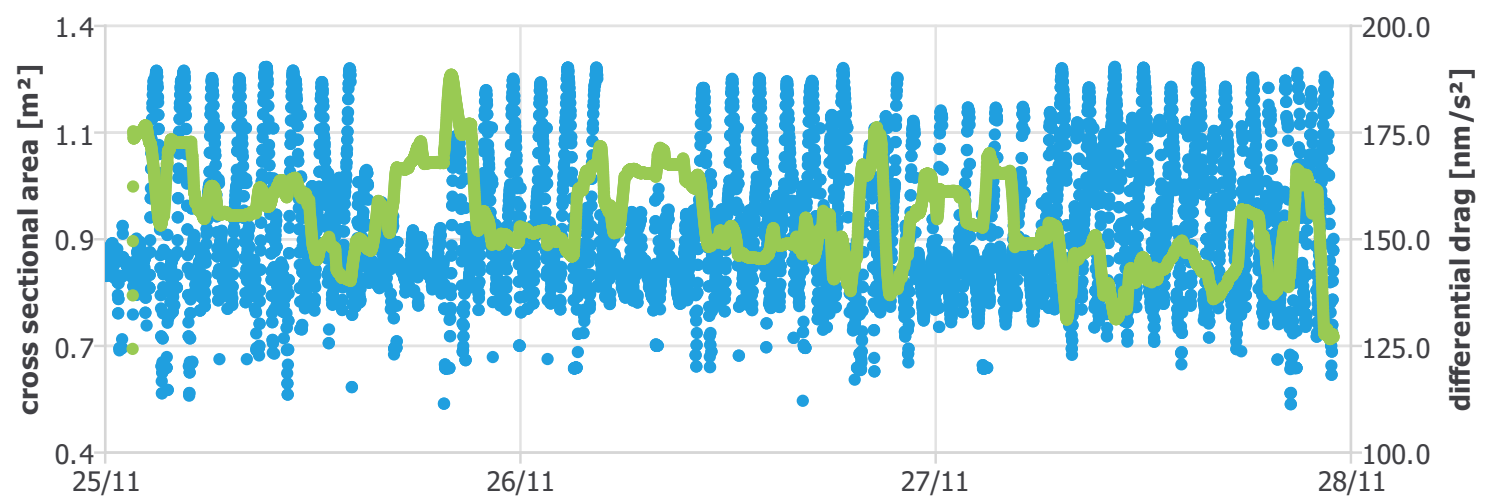

Figure 12. Cross-sectional area and differential drag at mid to close range

- Given the limited field of view of the camera $\left(12^{\circ} \times 18^{\circ}\right)$, a precise real-time knowledge of the relative motion is necessary to point the camera in the proper direction. In view of the aforementioned difficulties (inaccurate measurements and large perturbations of the onboard 
relative motion model), it becomes a real challenge to point properly the camera towards the target. Figure 13 depicts the real-time errors of the estimated direction to the target. From November $27^{\text {th }}$, the errors become so large that the camera points in the wrong direction, making impossible any measurement update anymore and leading eventually to a filter divergence. The fact that images are taken only every 30 seconds constitutes one of the major limitations of the navigation system. It would have probably been more robust (if the satellite would have allowed it) to work at a higher frequency (e.g. $1 \mathrm{~Hz}$ ) and to implement a simple attitude feedback controller to always keep the bright object in the center of the field of view, so that the navigation filter would have always been fed with measurements. ${ }^{17}$

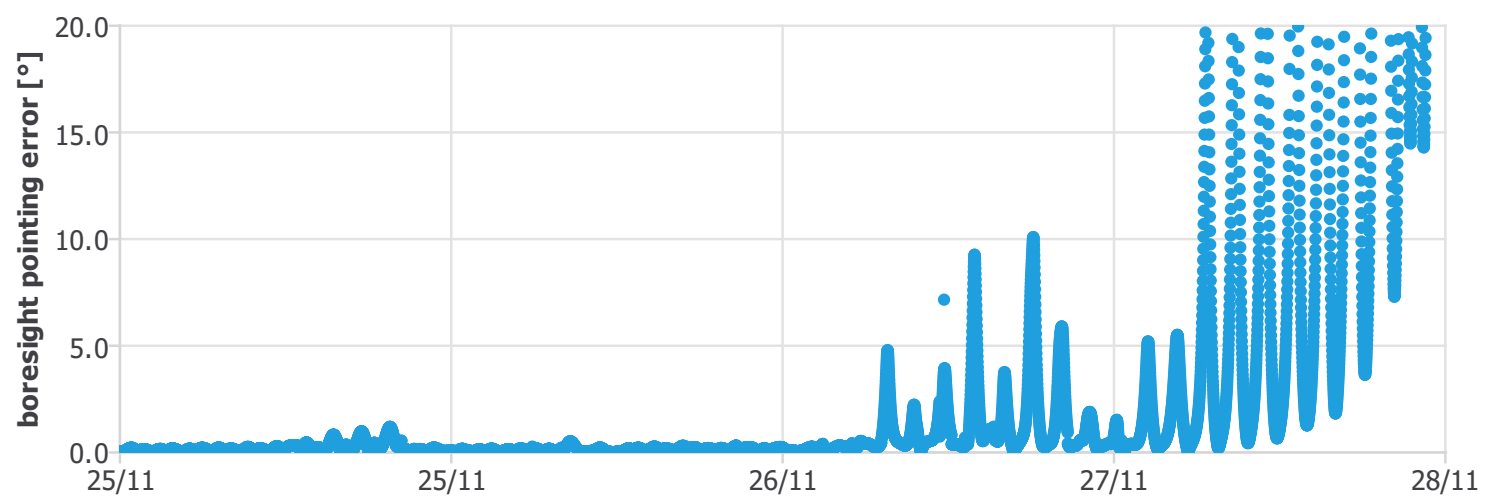

Figure 13. Onboard estimation errors of the direction to the target

Despite all these difficulties, the filter was able support the onboard guidance and control throughout the entire approach from $3 \mathrm{~km}$ to $50 \mathrm{~m}$, and could deliver a reliable navigation solution for some orbits at close range, yielding fantastic pictures of BEESAT-4. Fig. 14 depicts the achieved intersatellite separation and the time at which measurements were available (red dots). The blue dots correspond to the time of the four images. Note how the rectangular shape and the antennas of the

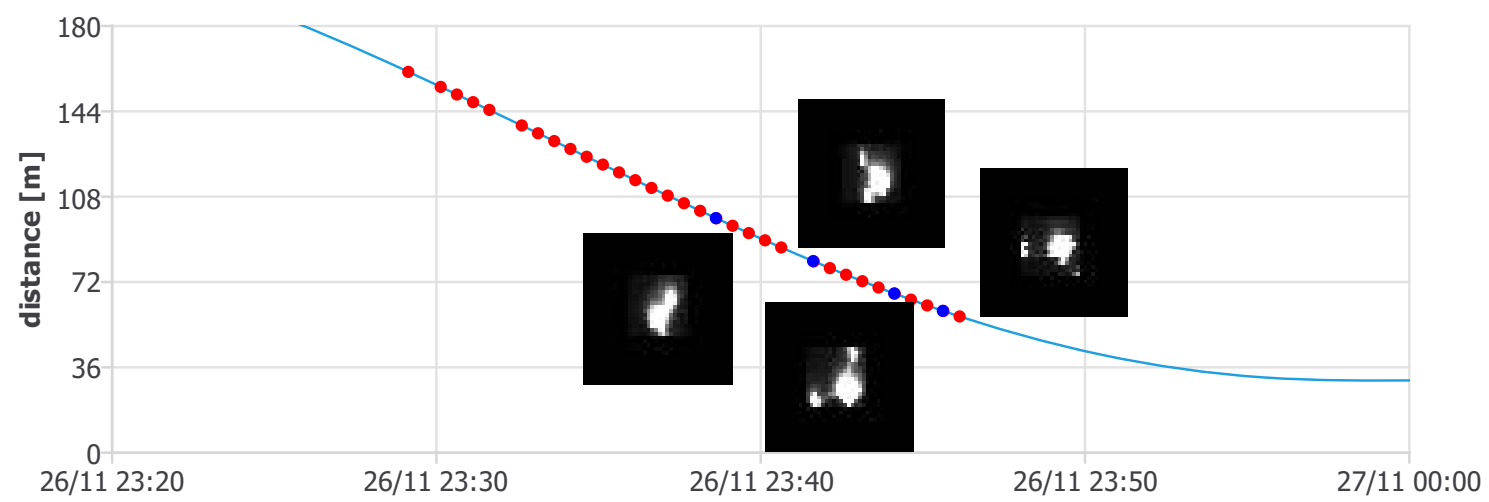

Figure 14. Measurements during the close range approach

spacecraft can be cleary recognized! In a mission dedicated to close-proximity operations, this corresponds exactly to the range where other kinds of metrology can be used (pose estimation, stereo vision, radar,...). Future investigations will focus on improving the robustness of the filter at close range but, considering the fact that the navigation system was not designed to work at that range, 
it can be already stated that the onboard autonomous rendezvous software based on line-of-sight navigation fullfiled brillantly its mission: bringing the separation down to less than $100 \mathrm{~m}$ !

\section{CONCLUSION}

For the first time in space history, the capability to approach autonomously in orbit a truly noncooperative object using a single camera has been demonstrated. This achievement was made possible thanks to the real-time angles-only relative navigation system, which could successfully support the onboard guidance and control tasks to perform smooth and safe rendezvous up to $50 \mathrm{~m}$ distance, paving the way to future close-proximity operations.

\section{REFERENCES}

[1] G. Gaias, J.-S. Ardaens, and C. Schultz, "The AVANTI experiment: flight results," Salzburg, Austria, $10^{\text {th }}$ International ESA Conference on Guidance, Navigation \& Control Systems, 2017.

[2] W. Halle, W. Bärwald, T. Terzibaschian, M. Schlicker, and K. Westerdorf, "The DLR -Satellite BIROS in the Fire-Bird Mission," Porto Petro, Majorca, Spain, Small Satellites Systems and Services Symposium, 2014.

[3] F. Baumann, S. Trowitzsch, and K. Brieß, "BEESAT - A CubeSat Series Demonstrates Novel Picosatellite Technologies," Brussels, Belgium, $4^{\text {th }}$ European CubeSat Symposium, 2012.

[4] S. Roemer and S. Stoltz, "SPL Light Weight Deployment Mechanism for Single CubeSats and DPL for Double CubeSats," Funchal, Madeira, Portugal, Symposium on Small Satellite Systems and Services (4S), 2010.

[5] S. D’ Amico, J.-S. Ardaens, G. Gaias, H. Benninghoff, B. Schlepp, and J. L. Jørgensen, "Noncooperative Rendezvous Using Angles-Only Optical Navigation: System Design and Flight Results," Journal of Guidance, Control, and Dynamics, Vol. 36, No. 6, 2013, pp. 1576-1595. doi: 10.2514/1.59236.

[6] G. Gaias, J.-S. Ardaens, and T. Terzibaschian, "Paving the Way for Future On-Orbit-Servicing Missions: the AVANTI Experiment," Munich, Germany, $25^{\text {th }}$ International Symposium on Space Flight Dynamics ISSFD, 2015.

[7] S. Weiß, F. Kempe, and K. Brieß, "GPS Tracking on the Three-Axis-Stabilized Picosatellite BEESAT4," Würzburg, Germany, 2013. $7^{\text {th }}$ Pico and Nano Satellite Workshop on Technology for Small Satellite Research.

[8] J.-S. Ardaens and G. Gaias, "Spaceborne Autonomous Vision-Based Navigation System for AVANTI," Toronto, Canada, 65 ${ }^{\text {th }}$ International Astronautical Congress, 2014.

[9] D. C. Woffinden and D. K. Geller, "Observability Criteria for Angles-Only Navigation," IEEE Transactions on Aerospace and Electronic Systems, Vol. 45, No. 3, 2009, pp. 1194-1208.

[10] J.-S. Ardaens and G. Gaias, "Angles-Only Relative Orbit Determination during the AVANTI Experiment," Matsuyama, Japan, 26 ${ }^{\text {th }}$ International Symposium on Space Flight Dynamics ISSFD, 2017.

[11] G. Gaias, S. D' Amico, and J.-S. Ardaens, "Angles-Only Navigation to a Noncooperative Satellite Using Relative Orbital Elements," Journal of Guidance, Control, and Dynamics, Vol. 37, No. 2, 2014, pp. 439451. doi: 10.2514/1.61494.

[12] O. Montenbruck, M. Kirschner, S. D'Amico, and S. Bettadpur, "E/I-Vector Separation for Safe Switching of the GRACE Formation," Aerospace Science and Technology, Vol. 10, No. 7, 2006, pp. 628635. doi: 10.1016/j.ast.2006.04.001.

[13] J.-S. Ardaens and S. D'Amico, "Spaceborne Autonomous Relative Control System for Dual Satellite Formations," Journal of Guidance, Control, and Dynamics, Vol. 32, No. 6, 2009, pp. 1859-1870.

[14] S. D'Amico, J.-S. Ardaens, and R. Larsson, "Spaceborne Autonomous Formation-Flying Experiment on the PRISMA Mission," Journal of Guidance, Control, and Dynamics, Vol. 35, No. 3, 2012, pp. 834850. doi: $10.2514 / 1.55638$.

[15] G. Gaias, J.-S. Ardaens, and O. Montenbruck, "Model of J2 Perturbed Satellite Relative Motion with Time-Varying Differential Drag," Celestial Mechanics and Dynamical Astronomy, Vol. 123, No. 4, 2015, pp. 411-433. doi: 10.1007/s10569-015-9643-2.

[16] G. Gaias and J.-S. Ardaens, "Design Challenges and Safety Concept for the AVANTI Experiment," Delft, The Netherlands, $8^{\text {th }}$ International Workshop on Satellite Constellations and Formation Flying, 2015. 
[17] G. Gaias and J.-S. Ardaens, "In-Orbit Experience and Lessons Learned from the AVANTI Experiment," University of Colorado Boulder, USA, $9^{\text {th }}$ International Workshop on Satellite Constellations and Formation Flying, 2017. 